\title{
Unusual case of Invasive Cribriform Carcinoma of Breast (ICC)
}

\author{
Swati Mishra, Kusum Yadav, Anshul Singh, Vatsala Misra, Kuldeep Chaudhary \\ Department of Pathology, Moti Lal Nehru Medical College, Prayagraj, Uttar Pradesh, India.
}

\section{Corresponding Author:}

Dr Swati Mishra

Email: drswatimishra456@gmail.com

This is an Open Access article distributed under the terms of the Creative Commons Attribution License (creativecommons.org/ licenses/by/3.0).

Received Accepted

Published

September 11, 2020

November 1, 2020

January 25, 2021

\begin{abstract}
Background: Invasive cribriform carcinoma (ICC) is one of the rare variants of invasive ductal carcinoma of breast accounting for $0.3 \%-0.8 \%$ of these cases. It has low malignant potential with nodal metastases or vascular invasion hardly ever seen and is therefore known to carry an excellent prognosis. Case Report: We report here a case of 58 year old lady who presented as a post-operative case of carcinoma left breast with recurrence after lumpectomy. The histopathology showed a dominant cribriform pattern of monomorphic tumor cells along with extensive perineural infiltration and lymphovascular invasion that prompted us to think of it initially as an adenoid cystic carcinoma (ACC) but was later corroborated as an ICC on immunohistochemistry. Conclusion: To the best of our knowledge and search of literature, this is the first case report of an ICC portraying such an aggressive behaviour.
\end{abstract}

Keywords: Adenocarcinoma, Adenoid Cystic Carcinoma, Breast Neoplasms, Ductal Carcinoma. Mastectomy.

\section{Introduction}

Invasive cribriform carcinoma (ICC) is quite a rare type of breast cancer with an incidence of $0.3-0.8 \%$ [1]. On histopathology, angulated islands with well-defined cribriform spaces, formed by arches of small to medium sized cells displaying minimal pleomorphism are seen. Micro-calcifications can also be present within the lumina [1]. Overall ICCs are said to carry a very good prognosis [2-5]. The commonest differential diagnosis is adenoid cystic carcinoma due to morphological similarity [1]. We present here an unusual case of an aggressive ICC breast in an elderly female that prompted us to consider it as an adenoid cystic carcinoma (ACC) initially.

\section{Case Report}

A 58 years old female presented to the Surgery outpatient department as a follow up post-operative case of carcinoma left breast with recurrence after lumpectomy. Post-lumpectomy patient had also received radiotherapy and chemotherapy, the details of which were not available. There was no significant family history of breast lump or hormonal therapy intake by the patient. CT thorax revealed a small dense nodule in left breast (? recurrence) along with few enlarged left axillary lymph nodes (? metastasis). Her routine haematological investigations were within normal limits. She was referred to the Oncosurgery department for further management where a left sided modified radical mastectomy (MRM) was done and the specimen was sent to us for histopathological examination.

The MRM specimen measured $18 \times 14.5 \times 5 \mathrm{~cm}$. Nipple areola complex (NAC) was not identified grossly due to previous lumpectomy. On further sectioning, a grey brown cystic area (tumor) measuring $2 \times 2 \times 1.5 \mathrm{~cm}$ in size was seen at the centre. All the margins and base were grossly free. A separate axillary tail received measured $3 \times 2 \times 1 \mathrm{~cm}$ from which 13 lymph nodes were dissected out. Sections from tumor, all four 
margins, base and dissected lymph nodes were taken and processed.

Sections examined from the tumor area displayed small clusters as well as scattered atypical cells in vast pools of mucin. These atypical cells had high $\mathrm{N}: \mathrm{C}$ ratio, hyperchromatic nuclei with scant to moderate amount of eosinophilic cytoplasm. At many places these tumor cells were showing a prominent cribriform pattern of arrangement with regular punched out spaces filled with eosinophilic material [Fig.1]. In some of the lumen calcification was seen [Fig.2]. Extensive areas of perineural infiltration were noted [Fig.3]. One foci of vascular invasion was also seen [Fig.4]. All margins and base were free of tumor. Out of thirteen lymph nodes dissected, two showed infiltration by tumor cells [Fig.5]. On the basis of predominant cribriform pattern of tumor cells seen, a differential diagnosis of adenoid cystic carcinoma (ACC) and invasive cribriform carcinoma (ICC) was given. For confirmation, an IHC panel of ER, PR and CD117, was advised which exhibited strong ER and PR positivity and absolutely negative CD117 [Fig.6,7]. Thus a final diagnosis of invasive cribriform carcinoma with lymphovascular invasion and extensive perineural infiltration was given. The patient was lost to follow-up and hence the post-operative treatment given to her and her response to it cannot be ascertained.

\section{Discussion}

Breast cancer is the most frequent cancer among women worldwide with the 5 year prevalence being $38.2 \%$ in Asia [4]. Histopathological classification divides invasive carcinomas into invasive ductal carcinoma (IDC) and invasive lobular carcinoma (ILC), the former being far more common. Invasive cribriform carcinoma (ICC) is a rare variant of IDC with an incidence of $0.3-0.8 \%$ [1]. It is seen in elderly females and usually presents on radiology as an occult mass. On microscopy it is arranged as invasive, often angulated islands with well-defined cribriform spaces, formed by arches

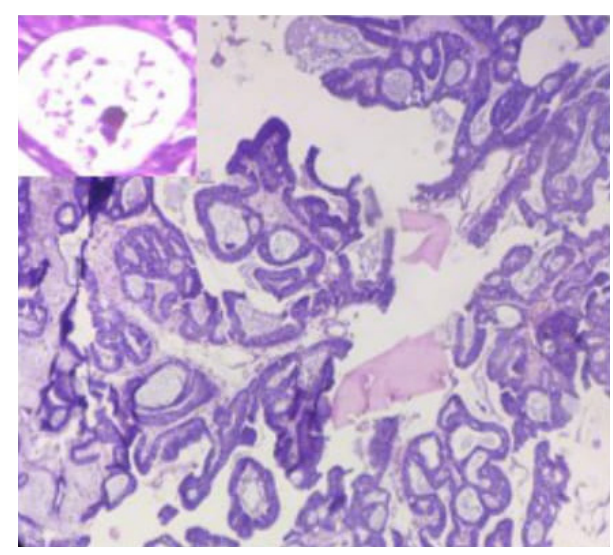

Fig.1: Tumor depicting a prominent cribriform pattern (H $\& E \times 4)$. Inset image showing calcification in the lumen.

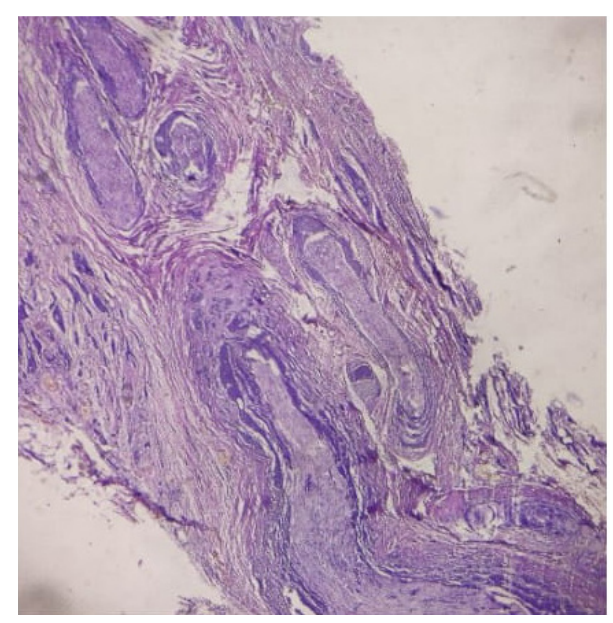

Fig.2: Extensive perineural infiltration by the tumor cells $(H \& E \times 4)$.

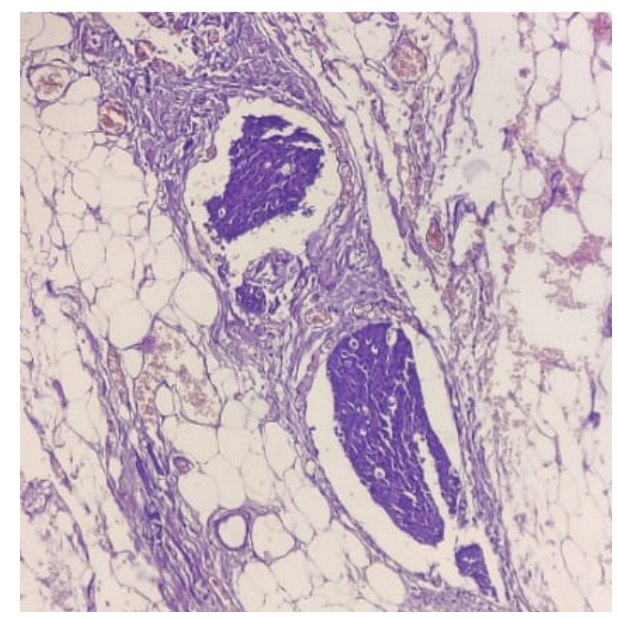

Fig.3: Foci of vascular emboli $(H \& E \times 40)$. 
of small to medium sized cells tumor cells arranged in sieve like pattern showing little pleomorphism. Mucin positive secretions bearing microcalcifications can often be seen within the lumina [1]. ICC is considered a malignant neoplasm with good prognosis attributed to the low frequency of axillary nodal metastases as well as vascular invasion $[2,6,7]$. The immunohistochemical studies show strong estrogen receptor (ER) and progesterone receptor (PR) positivity, whereas human epidermal growth factor receptor 2 (HER2) amplification is rarely observed $[2,4,5]$. Reckoning the low malignant potential, conservative surgery is considered as the primary treatment for ICCs. Endocrine therapy is significant because the majority of tumors are positive for ER/ PR. In our case, we witnessed a predominant cribriform pattern of the tumor cells. Though the N:C ratio was not very high, foci of lymphovascular invasion and extensive perineural infiltration seen averted our diagnosis more towards adenoid cystic carcinoma. However, the IHC findings confirmed it as an ICC. A search for literature to look for any similar case reports of aggressive ICCs was negative.

ICCs bear quite a histological resemblance to adenoid cystic carcinomas (ACCs) which becomes its closest differential. ACCs are rare, comprising $<0.1 \%$ of breast carcinomas with a predilection for sub-areolar region. On histopathology, they consist of a dual-cell population of luminal and myoepithelial-basal cells arranged in tubular or cribriform architecture with mucin filled lumina [6]. Distant metastases and regional lymph node involvement are rarely observed in ACCs except for the solid variant. The immunohistochemistry of ACC of the breast fits well with basal-like features, negative for ER, PR, and Her2 neu. CD 117 positivity which is acknowledged in ACCs of salivary gland is also very well flaunted by breast ACCs [1]. The treatment for it is mainly conservative surgery with axillary dissection advised only in high grade lesions [2]. The role of chemotherapy and radiotherapy is not well elucidated but the tumor is non-responsive to endocrine therapy [8].

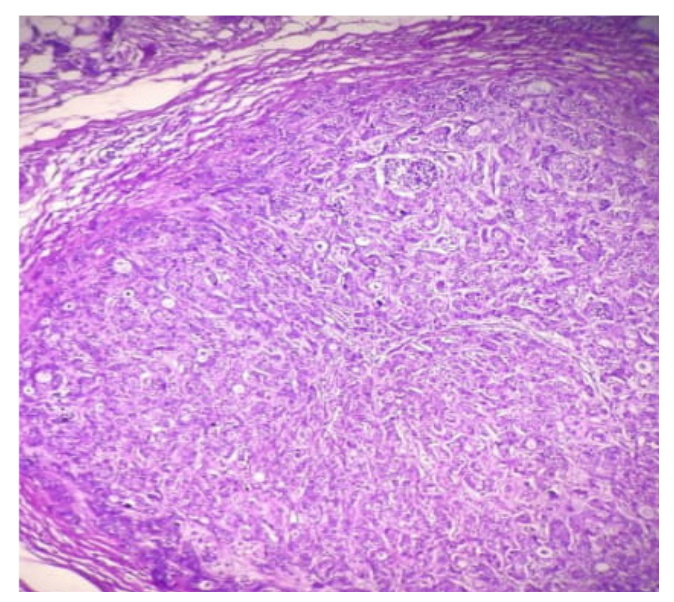

Fig.4: Invasion of lymph node by tumor cells ( $H \& E \times 40)$.

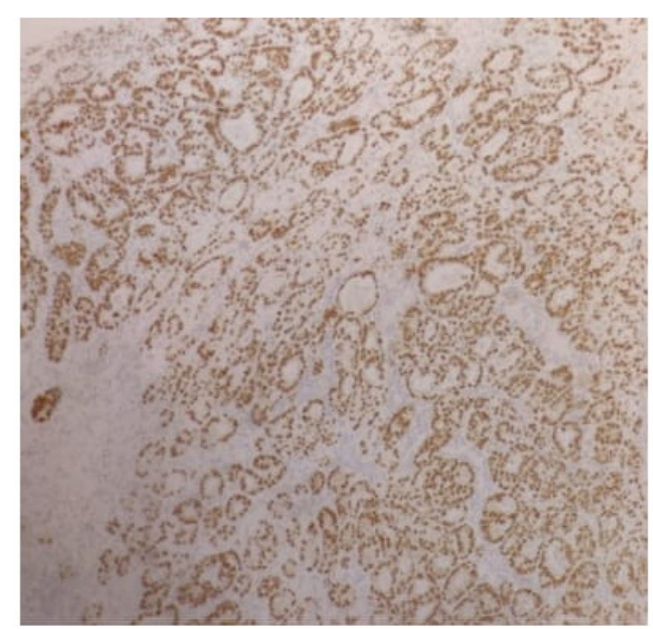

Fig.5: Tumor cells showing diffuse ER positivity $(I H C \times 4)$.

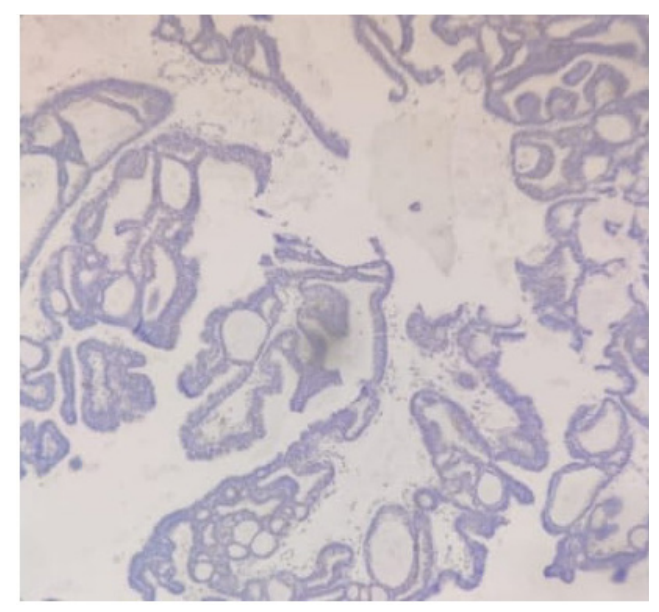

Fig.6: Tumor cells negative for CD117 (IHC $\times 4)$. 
Besides ACCs another differential to be considered is secretory carcinoma (SC) [9]: translocation associated $[\mathrm{t}(12 ; 15)]$, new and a rare invasive carcinoma with incidence of less than $0.15 \%$ and median presentation age of 25 years. Three patterns are seen: microcystic, tubular and solid, out of which the tubular pattern may mimic an ICC. The bespeak of SC is the presence of intracellular and extracellular secretory material that is PAS/Alcian blue positive. It is also triple negative on IHC, but frequently express alphalactoalbumin, while CD117 and E-cadherin are occasionally expressed. Though it has a favourable prognosis, an aggressive course is seen in older patients [1]. There is dearth of data on the treatment of secretory carcinoma. At present, conservative treatment in the form of surgical excision is recommended [9].

To conclude, ICC is a rare variant of invasive ductal carcinoma with an indolent behaviour and excellent prognosis. In our case however we witnessed a disparate finding of an ICC showing extensive perineural infiltration along with lymphovascular invasion. In all likelihood and to the best of our search for literature, this is the first case report of an ICC showing an aggressive behaviour. The parallelism of histological features between ICCs and ACCs makes it necessary to differentiate between the two because of difference in their management with respect to endocrine therapy.

\section{Conclusion}

Invasive cribriform carcinoma (ICC) breast is a rare entity of invasive ductal carcinoma. It has low malignant potential with metastasis barely encountered and therefore has an excellent prognosis. We present here a case report on ICC breast depicting aggressive behaviour.
Contributors: SM: Histopathology and preparation of draft manuscript; KY, AS: histopathology and critical inputs into the manuscript; VM, KC: manuscript revision. SM acted as study guarantor. All authors approved the final version of the manuscript and are responsible for all aspects of the study. Funding: None; Competing interests: None stated.

\section{References}

1. Rakha E, Pinder SE, Shin SJ, Tsuda H. Tubular carcinoma and cribriform carcinoma. In: Lakhani SR, Ellis IO, Schnitt SJ, Tan PH, van de Vijver MJ, editors. WHO classification of tumours of the breast. $4^{\text {th }}$ edition. Lyon: IARC Press; 2012. pp. 43-45.

2. Venable JG, Schwartz AM, Silverberg SG. Infiltrating cribriform carcinoma of the breast a distinctive clinicopathologi centity. Hum Pathol. 1990;21:333-338.

3. Marzullo F, Zito FA, Marzullo A, Labriola A, Schittulli F, Gargano G, et al. Infiltrating cribriform carcinoma of the breast, a clinicopathologic and immunohistochemical study of five cases. Eur J Gynaecol Oncol. 1996;17:228231.

4. Park K. Epidemiology of Chronic Non-communicable diseases and conditions. In: Park K, editors. Park's Textbook of Preventive and Social Medicine, $25^{\text {th }}$ ed. Madhya Pradesh: Banarasidas Bhanot; 2019. pp. 418.

5. Colleoni M, Rotmensz N, Maisonneuve P, Mastropasqua MG, Luini A, Veronesi P, et al. Outcome of special types of luminal breast cancer. Ann Oncol. 2012;23:14281436.

6. Page DL, Dixon JM, Anderson TJ, Lee D, Stewart HJ. Invasive cribriform carcinoma of the breast. Histopathology 1983; 7:525-536.

7. Rosen PP, Oberman HA: Invasive carcinoma. In: Tumors of the mammary gland (Atlas of tumor pathology, $3^{\text {rd }}$ series, fascicle 7). Armed Forces Institute of Pathology, Washington,D.C., 1993; pp. 157-257.

8. Coates JM, Martinez SR, Bold RJ, Chen SL. Adjuvant radiation therapy is associated with improved survival for adenoid cystic carcinoma of the breast. J Surg Oncol. 2010;102:342-347.

9. Horowitz DP, Sharma CS, Connolly E, Gidea-Addeo D, Deutsch I. Secretory carcinoma of the breast: results from the survival, epidemiology and end results database. Breast. 2012;21:350-353. 